\title{
Extraction of Water from Polar Lunar Permafrost with Microwaves - Dielectric Property Measurements
}

\author{
Edwin C. Ethridge \\ Marshall Space Flight Center, Huntsville, AL, 35812 \\ And \\ William Kaukler. ${ }^{\dagger}$ \\ University of Alabama in Huntsville, Huntsville, AL, 35899
}

\begin{abstract}
Remote sensing indicates the presence of hydrogen rich regions associated with the lunar poles. The logical hypothesis is that there is cryogenically trapped water ice located in craters at the lunar poles. Some of the craters have been in permanent darkness for a billion years. The presence of water at the poles as well as other scientific advantages of a polar base, have influenced NASA plans for the lunar outpost. The lunar outpost has water and oxygen requirements on the order of 1 ton per year scaling up to as much as 10 tons per year. Microwave heating of the frozen permafrost has unique advantages for water extraction. Proof of principle experiments have successfully demonstrated that microwaves will couple to the cryogenic soil in a vacuum and the sublimed water vapor can be successfully captured on a cold trap. The dielectric properties of lunar soil will determine the hardware requirements for extraction processes. Microwave frequency dielectric property measurements of lunar soil simulant have been measured.
\end{abstract}

\footnotetext{
*AST Ceramics Engineer, Materials \& Processes Lab., BIdg. 4600 EM40, AIAA non-Member.

${ }^{\dagger}$ Associate Professor, Department of Chemistry, AIAA nonmember.
} 
The Clementine remote sensing lunar satellite radar findings combined with Lunar Prospector's Neutron Spectrometer results provide evidence that there are significant quantities of hydrogen at the poles of the moon. Actually, water-ice is probably the source, being cryogenically trapped in the permanently shadowed craters at both lunar poles. It is likely of cometary origin; the same as for the oceans of Earth. Water will be an extremely valuable resource on the moon, not only for human habitation but also as propellant produced by electrolysis. It has been stated that there could be up to 200 million to billions of tons of water at the lunar poles. Paul Spudis, one of the Clementine Scientists has said that the poles of our moon could be, "the most valuable real estate in the solar system." Lunar water would likely be the first in situ resource (other than solar energy) to be exploited for human exploration making the concept a paradigm shift away from resupply towards local acquisition of water and oxygen. Living off the land could become more of a reality.

Microwave heating has the unique advantage for extraction of water because it heats from the inside. By delivering the microwave energy to where the water is, excavation would be nearly eliminated. Also, we would not have to "strip mine" the moon to extract the water. Since the microwave penetration depth is dependent on frequency, measurements of the dielectric properties of lunar regolith simulant permit the calculation of the penetration depth that can be used to maximize the efficiency of operations. The methods for water extraction would be directly applicable to Mars because of its rarefied atmosphere.

\section{Scientific Goals and Objectives}

The objective of this research is to further develop facilities, hardware, and methods testing water extraction methods from lunar permafrost simulant. Microwaves heat the regolith sublime causing the water ice to sublime and permitting its subsequent collection on a chilled surface on the lunar surface. Measurement of the dielectric properties of regolith simulant and actual lunar soil need to be measured to validate microwave penetration measurements. Dielectric property measurements at different wavelengths and to cryogenic temperatures will permit the design of microwave extraction schemes. Comparison of microwave dielectric properties with actual lunar soil can be used to evaluate the adequacy of lunar simulants for prototype experiments. The frequency dependence of microwave penetration into lunar regolith is needed for the design and optimal selection of microwave frequencies for lunar extraction. This investigation should permit the development of strategies for maximizing scientific and lunar resource yield from lunar surface activities.

\section{Proof of Principle Experiments}

A number of possible methods have been suggested for the extraction of water from lunar permafrost. Microwave processing has several very significant advantages over all other technologies. The use of solar energy immediately comes to mind. The thermal conductivity of lunar soil as measured at the Apollo 15 and 17 sites is extremely low. Comparisons against thermal conductivities of other materials revealed that lunar regolith has similar thermal conductivity to silica aerogel, a superinsulator. Heat at the surface will not penetrate deeply into the soil making solar heating unworkable. For example, the temperature 1 centimeter $(<1 / 2$ inch) beneath the surface heated to $1000 \mathrm{C}$ will reach $600 \mathrm{C}$ only after 40 hours by thermal conduction through the lunar regolith. Excavation of the soil and heating in a chamber is a possibility, but this would require excavation equipment, extra infrastructure, and could produce large quantities dust. The permafrost is at cryogenic temperatures and it has been reported that soil containing water at those temperatures can be as hard as granite. Excavation could be greatly minimized by microwave heating for water extraction. It has been reported from simple observations, that lunar regolith couples with microwave energy very well'. For this reason the Lunar Simulant Workshop ${ }^{2}$ identified the microwave properties of lunar soil as being an important property to be replicated with lunar simulants. Coupling of RF energy to a material depends upon the dielectric constant and the loss tangent. Remote sensing measurements of lunar regolith have been reported in the low RF range $10^{2}-10^{7} \mathrm{~Hz}^{3,4,5}$ some in $9.3 \mathrm{GHz}^{6}$ and many above $10 \mathrm{GHz}$ ${ }^{3}$, but to our knowledge there are no laboratory measurements of the complex dielectric permittivities and magnetic permeabilities in the 1 to $10 \mathrm{~Hz}$ range. Coincidentally, this is the same frequency range where microwave heating of lunar regolith might be performed with greatest efficiency. Microwave absorption in regolith is relatively optimal in this frequency range while simultaneously heating to depths on the order of a meter. Commercially available magnetrons provide the highest conversion efficiency and package compactness. Magnetron tube technology is only 
available within this frequency range. Five years ago, we began proposing the investigation of microwave processing of lunar regolith and water removal. Our first funding was under a MSFC lunar habitation study investigated the melting and sintering of lunar regolith simulant and began the development of methods for water extraction?. A MSFC Call for Proposal project funded a proof of principal experiment that measured the processing parameters for the extraction of water from lunar permafrost simulant in vacuum at cryogenic temperatures ${ }^{8}$. Lunar conditions were simulated inside a microwave transparent quartz vessel containing the regolith permafrost simulant. More recently we continued the work with a task on a Center Strategic Investment project and this past

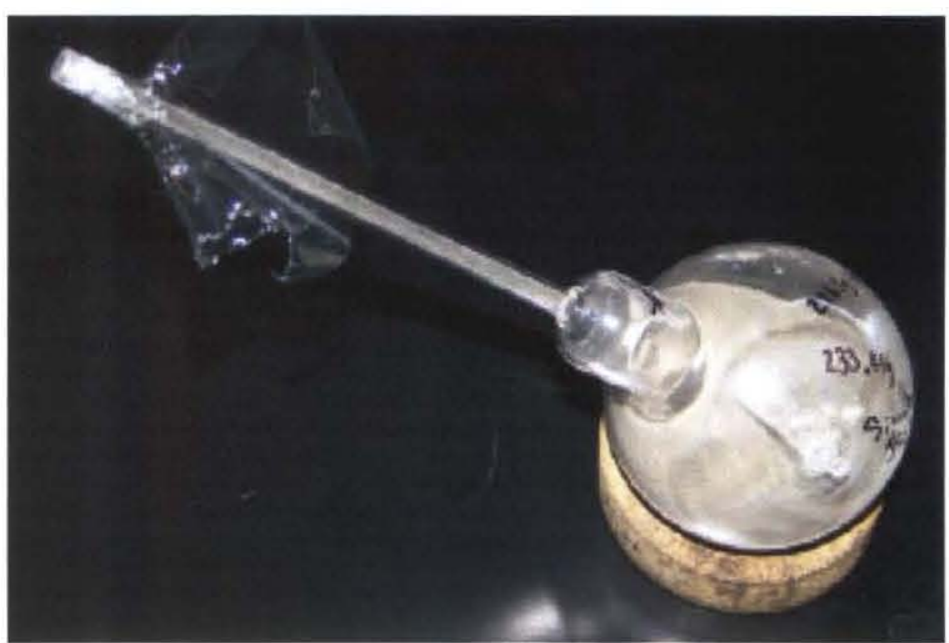

Figure 1. Quartz vessel containing lunar regolith simulant permafrost ( $1 \%$ water). summer we were awaraded a NASA HQ ROSES 4 year project to further develop the technology.

Original plans were to set up a full scale experiment inside a large vacuum chamber. But, because of the limited resources throughout these projects, we started with an experiment that we refer to as the "moon in a bottle" (Figure 1). The permafrost simulant was prepared by adding the appropriate quantity of deionized water to regolith simulant contained in a quartz vessel and sealed (Figure 1). The simulant was previously dried under vacuum with microwave heat to ensure adsorbed moisture from the air was removed. Quartz was selected for the container since it is transparent to microwave radiation. Water-ice concentrations in the range of 0.5 to 2 weight $\%$ were examined. With the addition of water, the simulant initially clumped like wet sand. After several days in the sealed container at room temperature, the water spread uniformly throughout the simulant and it again flowed like sand. The regolith water mixture was mixed and the quartz vessel was placed into and supported by a bed of stimulant. Liquid nitrogen was poured over the vessel to freeze the water. The soil increased its "flowability" after freezing showing that ice particles behave like dry powder. 
A simple "proof of principle" lunar test bed experiment simulating the cryogenic temperatures, high vacuum, suitable simulant with representative quantities of water was designed and tested. The quartz vessel containing simulated lunar permafrost (JSC-1 with $1 \%$ water) was placed in a $1 \mathrm{~kg}$ bed of JSC-1 lunar simulant modeling the lunar surface. This assembly was placed inside of a commercial $1 \mathrm{KW}, 2.45 \mathrm{GHz}$ microwave oven. See Figure 2. The quartz vessel was attached to a turbomolecular vacuum pumping station (Varian Turbo V200) with a liquid nitrogen cold trap in line to capture the extracted $\mathrm{H} 2 \mathrm{O}$ for yield data. Liquid nitrogen was slowly and repeatedly poured over the outside of the quartz flask into the regolith simulant bed to achieve temperatures in the range of $(-200$ to $-50 \mathrm{C})$. A vacuum level of 10-5 Torr was pumped on the quartz flask and monitored with a Pirrani gage. At temperatures below $-50 \mathrm{C}$, the water vapor pressure is less than this vacuum level, so water was not lost from the vessel. Power to the microwave was applied in 30 second steps. Between steps when the power was off, temperature measurements were quickly made by a thermocouple

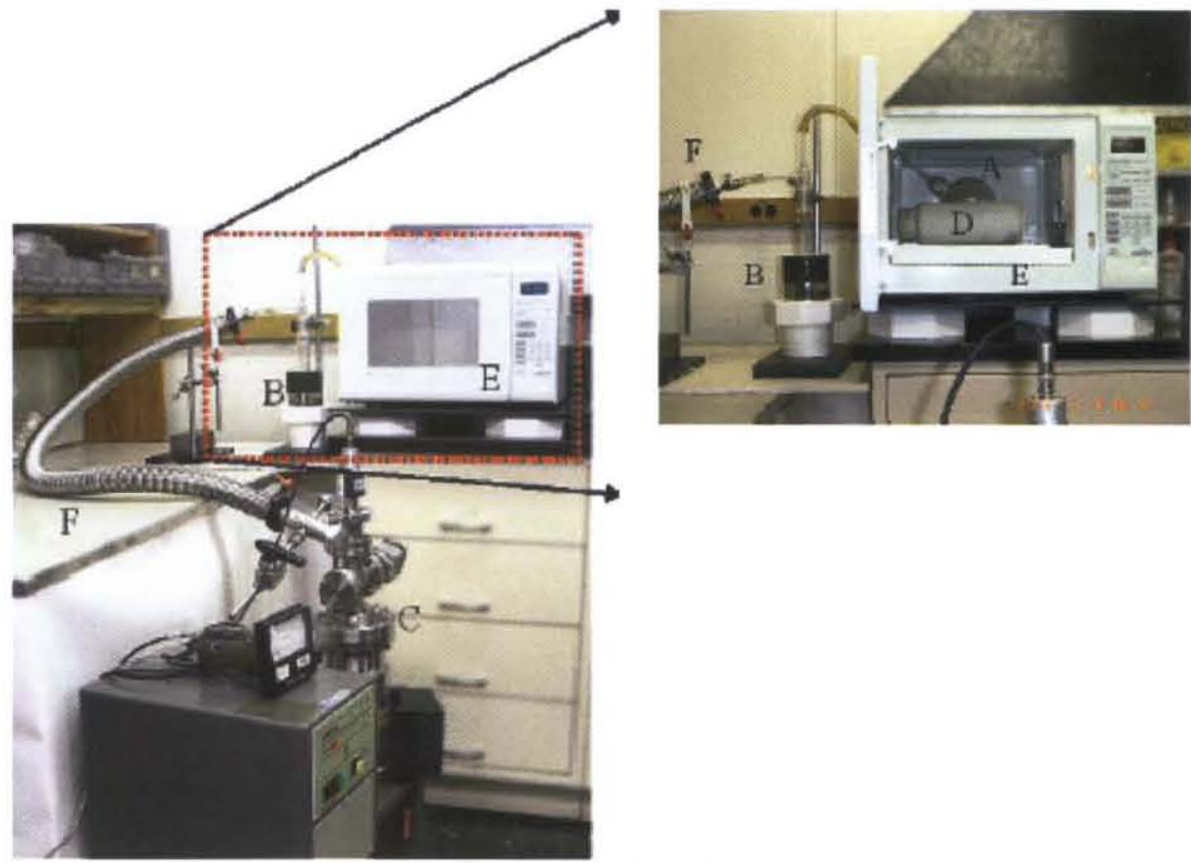

Figure 2. Laboratory setup for the proof of principle experiments. The quartz vacuum vessel (A) rests in a regolith bed contained by a polyethylene container (D) inside a commercial microwave oven. A vacuum line attached to the quartz vessel (A) contains a cold trap (B) in line with the turbo-molecular vacuum pumping station (C). placed into the thermowell in the quartz vessel and inserted into the regolith simulant bed. The experimental data consisted of temperature, vacuum level, time, and simulant weights before and after processing. When the permafrost simulant was between $50 \mathrm{C}$ and $0 \mathrm{C}$, the water vapor pressure in the vessel was proportional to the temperature but less than 0.0002 Torr. The pressure suddenly jumped to 0.001 Torr after a couple of minutes of microwave exposure when the simulant temperature rose above $0 \mathrm{C}$. Examination of the cold trap showed most of the water ice was collected before the temperature reached $0 \mathrm{C}$. This demonstrates that water was extracted by sublimation directly from ice at permafrost temperatures well below the freezing point of water. The JSC-1A simulant coupled very well with $2.45 \mathrm{GHz}$ microwave energy. With a $\sim 1 \mathrm{KW}$ power source, $1 \mathrm{~kg}$ of JSC-1A increased from $-100 \mathrm{C}$ to $-100 \mathrm{C}$ in 2 minutes. The water rapidly sublimes when irradiated with microwave energy. Then the water vapor in the vacuum is efficiently captured by a liquid nitrogen cold trap. At the lunar pole the cold trap can be easily chilled below $-50 \mathrm{C}$. Greater than $95 \%$ of the original water content in the permafrost was removed by sublimation during $\sim 2$ minutes of processing. Of this extracted water, $>98 \%$ was captured in the cold trap. The results from these tests with our simulated lunar vacuum cryogenic test bed facility conclusively verified the microwave water extraction concept. A $1 \mathrm{KW}$ microwave removed $2 \mathrm{~g}$ of water from $200 \mathrm{~g}$ of simulant at cryogenic temperature. But, the $1 \mathrm{Kg}$ simulant bed was also heated. An additional $10 \mathrm{~g}$ of water also could have been extracted. An engineering metric for the process is that more than $2 \mathrm{~g}$ and up to $12 \mathrm{~g}$ of water can be extracted in 2 minutes with $1 \mathrm{KW}$ of power. In one hour one could expect between 60 and $360 \mathrm{~g}$ of water might be extracted. Water is required for a human outpost on the moon. Work on the International Space Station has demonstrated that water for human habitation can be recycled with processes such as ECLIS, but they are not $100 \%$ efficient. It is estimated that on the lunar outpost ECLIS should be $95 \%$ efficient. This leads to the lunar architecture study requirement that 1 metric ton of water per year will be required by 2023 for the habitat. The extraction of one metric ton of water per year using $1 \mathrm{KW}$ of power is within the bounds of these numbers. The microwave water extraction process, therefore, is a very viable method for water extraction as per lunar architecture study requirements. 


\section{Dielectric Property Measurements}

Microwave heating occurs by the absorption of the energy in the material, with the power decaying exponentially with distance into a material. In dielectric materials, RF radiation of millimeter or longer wavelengths can penetrate meters and even hundreds of meters into the soil depending on the frequency. The absorption of the microwave energy is dependent on the complex dielectric constant and wavelength.

\section{A. Background}

The response to an electric field caused by the material complex electric permittivity (complex dielectric constant) and to a lesser degree, the response to the magnetic permeability (real and imaginary relative permeability), are critical to understanding the interaction of microwaves with the regolith and the design of microwave heating and processing systems. As far as we know, no electric permittivity measurements have been made in the frequency range of interest and no magnetic permeability measurements of any kind have ever been made of regolith simulant materials and actual lunar soil.

The absorption of microwave energy is simply expressed as being directly related to frequency, and the root of the dielectric constant and loss tangent (see Table I). This is most easily visualized with the nomograph in Figure 3, is a plot of dielectric constant on the $\mathrm{x}$-axis and loss tangent on the $\mathrm{y}$-axis. Lines of constant (1/e) penetration depth diagonally cross the plot. Note the major difference between water (liquid) and ice (crystal) properties.

Table 1. Textbook Equations for Dielectric Properties and the Absorption Coefficient.and Power Absorbed,

$P$, as a Function of Depth, $z$.

$$
\begin{aligned}
& \frac{\partial P}{\partial z}=-2 \alpha P \\
& P(z)=P_{0} e^{2 a} \\
& \varepsilon^{*}=\varepsilon^{\prime}-i \varepsilon^{\prime \prime} \\
& \tan \delta_{e}=\varepsilon^{\prime \prime} / \varepsilon^{\prime} \\
& \alpha=\frac{2 \pi f}{c} \sqrt{\frac{\varepsilon^{\prime}}{2}}\left\{\sqrt{1+\tan ^{2} \delta_{c}-1}\right\} \\
& \varepsilon \text { real part of relative permittivity, } \\
& \varepsilon \text { " imaginary part of relative permittivity, } \\
& \tan \delta \text { loss tangent, and } \\
& \alpha \quad \text { absorption coefficient. }
\end{aligned}
$$

The Beer-Lambert Law expresses the exponential attenuation of radiation as it passes through a material in terms of the attenuation coefficient, $\alpha$, and distance into the material, $\mathrm{z}$.

$$
\mathrm{I}(\mathrm{z}) / \mathrm{Io}=1 /(\mathrm{e} \alpha \mathrm{z})
$$

Attenuation of the incident radiation intensity (irradiance), Io, $\mathrm{I} / \mathrm{Io}$ to equal $1 / \mathrm{e}(0.368)$, means that $\alpha \mathrm{z}=1$, and the skin depth, $\delta$, is,

Skin depth $=\delta=z=1 / \alpha$

This means that $63 \%$ of the intensity of radiation is absorbed within the skin depth. The language used to describe the attenuation and power absorbed by materials can be a source of confusion. The Beer-Lambert Las expresses the intensity of radiation, and in particular the intensity of the electric field. Engineering applications refer to the power absorbed. According to Jackson 10 the power absorbed is proportional to the electric field squared. Such that,

$\mathrm{P}(\mathrm{z}) / \mathrm{Po}=1 /(\mathrm{e} 2 \alpha \mathrm{z})$

and for attenuation of power to $1 / \mathrm{e}, 1=2 \alpha \mathrm{z}$, the penetration depth is,

Penetration depth $=z=1 / 2 \alpha$

Therefore, the depth at which the power is reduced to $1 / \mathrm{e}$ (63\% power absorbed) is half the normal skin depth. The units for penetration depth are $\mathrm{m} / \mathrm{Neper}$, for power reduced to $1 / \mathrm{e}$. The penetration depths calculated and plotted in nomograph in Figure 3 are for power absorbed. As the microwave energy penetrates regolith, its intensity is 
attenuated, being transformed to heat. Since the removal of water by sublimation is dependent upon heating of the regolith, all of the energy delivered into the regolith will contribute to the process of water extraction.

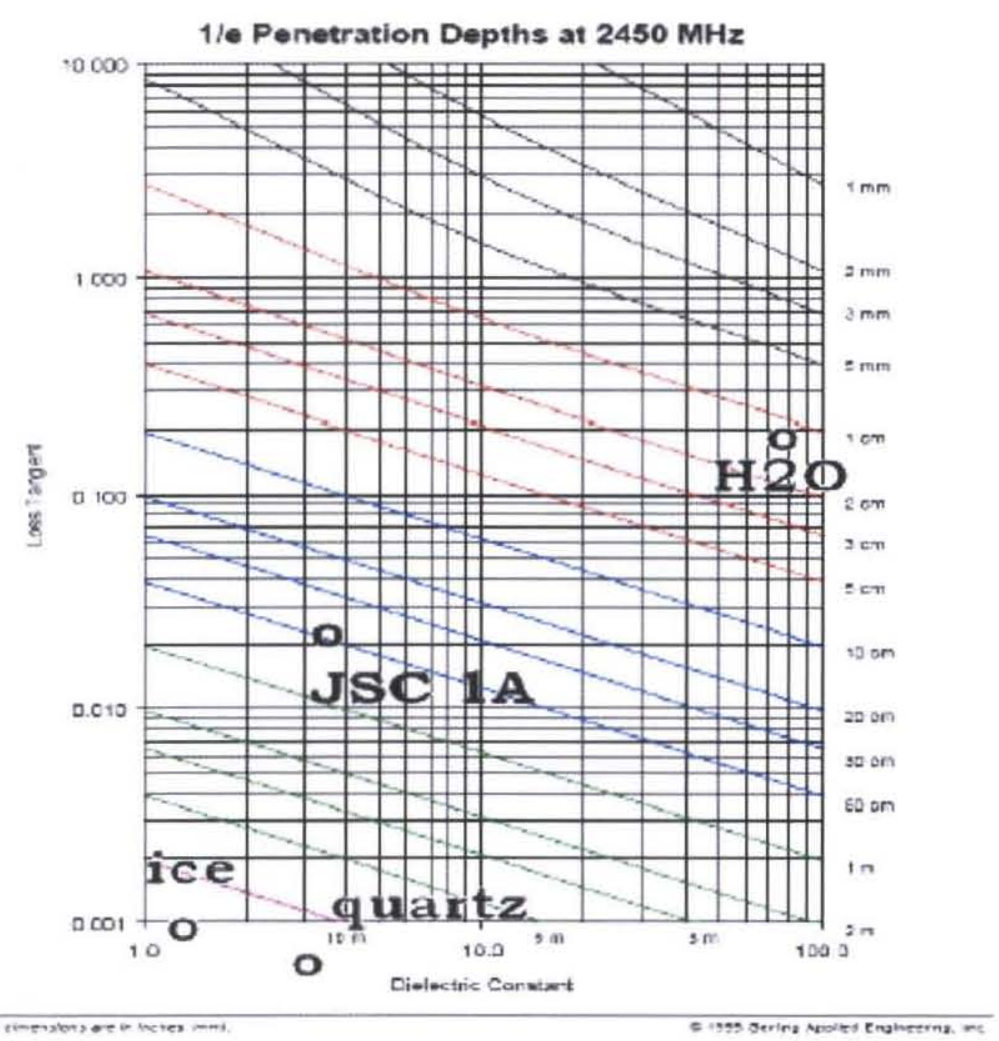

Figure 3. Nomograph that graphically illustrates the relationship between the dielectric constant, the loss tangent, at a given frequency and penetration depth (1/e of the initial microwave power) for materials of interest.

Our plan is to measure the complex dielectric properties of JSC-1, JSC-1a and other simulants at $12-8 \mathrm{GHz}, 2.2$ $-3.3 \mathrm{GHz}$, and $0.9 \mathrm{GHz}$ at room temperature. Measurements of JSC-la have been made at room temperature and at cryogenic temperatures. An pending application to the JSC lunar sample repository has requested a loan of lunar soil for property measurements at $2.2-3.3$ and $8-12 \mathrm{GHz}$ bands at room temperature and cryogenic temperature.

Lunar soil differs from the simulants in two important ways. The oxidation state of the iron oxide in lunar soil is as $\mathrm{FeO}\left(\mathrm{Fe}^{2+}\right)$. The terrestrial derived lunar regolith simulants contain a mixture of mostly $\mathrm{Fe} 2 \mathrm{O} 3\left(\mathrm{Fe}^{3+}\right)$ and little $\mathrm{FeO}\left(\mathrm{Fe}^{2+}\right)$. A number of Apollo regolith samples also contain significantly more TiO than the simulants. But, possibly the most significant difference is that Lunar samples contain small concentrations of metallic Fe. Two possible sources of the metallic iron are meteorites and "weathering" of the lunar soil grains. Micrometeorite impacts can locally flash heat the surface of grains of soil to above the melting temperature, causing the agglomeration of grains into what is called agglutinates. Examination of the lunar grains has shown that just under the surface is a layer containing metallic nanoparticles of $\mathrm{Fe}^{0}$. During the flash heating, atomic hydrogen from Solar winds can reduce the iron oxide in the surface of the grains to elemental iron. It has been postulated that this nanophase iron causes lunar soil to be strongly coupled to microwave energy. The Lunar Simulant Workshop in Huntsville (2004) concluded that a derived simulant from JSC-la should be developed. Orbitec Inc. has produced and provide to us samples of a nano-phase iron containing agglutinated simulant that was developed under an SBIR project. We are planning to make dielectric measurements in the near future on this simulant to compare with actual Apollo lunar soil. An important goal will be to determine if nano-phase $\mathrm{Fe}^{0}$ has a significant effect on the 
dielectric properties. This research will develop the methods to determine if there are significant measurable differences between the different lunar regolith simulants and Apollo lunar soil.

\section{B. Dielectric Measurement Methods and Facilities}

In this study we used "state of the art" methods for the measurement of the dielectric properties of lunar regolith simulants. The MSFC Microwave/Millimeter Instrumentation Facility (EM40) has the Agilent PNA series network analyzer (model number E8361A with A.06.04.30 firmware, Figure 4) that was used to measure the dielectric properties of the regolith simulants and lunar soil. A waveguide fixture was used to make the dielectric property measurements of simulants. A dedicated software package, Agilent 85071 version E2.24.02 Materials Measurement Software from Innovative Solutions Inc. takes the data from the analyzer and fits it to appropriate models to extract the desired electrical properties.

We selected the transmission line technique that uses a relatively small amount of sample. This is especially well suited for powdered regolith simulant and actual lunar soil samples. The limitations of the method are that each setup is limited to a small range of frequencies per test fixture, a larger quantity of sample is required for lower frequencies, precisely known sample geometries are required, and it works best with low magnetic susceptibility materials. This technique works by measuring the phase shifts during absorption and/or reflection of microwaves from both sample surfaces within a tuned waveguide (4 measured signals for data analysis). The measurements from the instrument are used with the different software models developed for the instrument. The models iteratively calculate the complex permittivity and/or permeability using the 4 measured signals. One of these models determines the

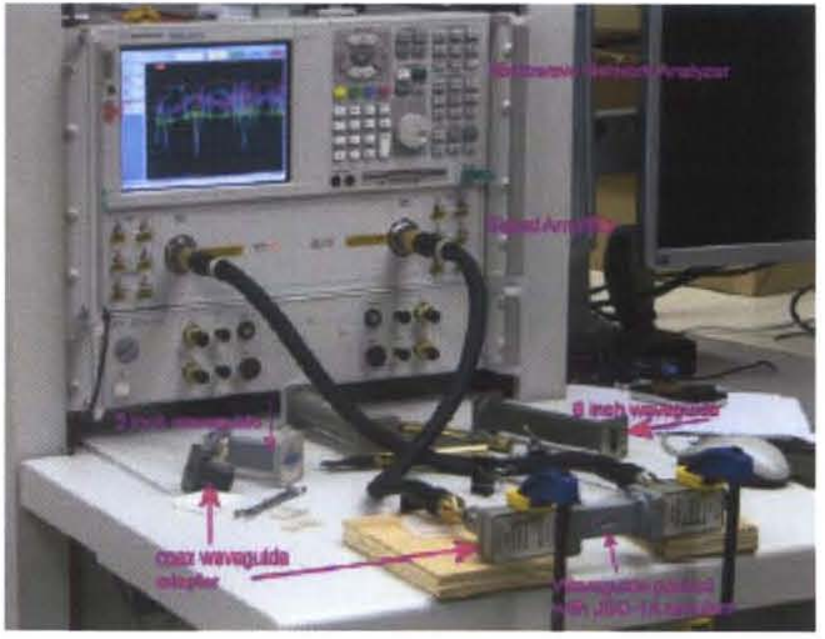

Figure 4. Agilent Network Analyzer and test assembly for microwave dielectric property measurements. complex magnetic permeability of the specimen along with complex permittivity.

The various dielectric deconvolution models available in the Agilent software package were evaluated. Two of the models are the 4-parameter permittivity model (no magnetic properties) and the 2-parameter, transmission only models (also no magnetic properties). By excluding the evaluation of the permeability properties, these two models produce slightly different permittivity results with additional irregularities that will not be discussed here.

Measurement cells for $10 \mathrm{GHz}$ range $(8-12$ $\mathrm{GHz}$ ) were developed in-house and tested first. Coax to microwave adapters and microwave flanges for $2.45 \mathrm{GHz}$ range $(2.2-3.3 \mathrm{GHz})$ and $0.9 \mathrm{GHz}$ were adapted for dielectric measurements with the network analyzer. The dielectric measuring apparatus for our $10 \mathrm{GHz}$ cell is shown in Figure 5. For all these waveguide materials measurements, since the simulant is in the form of a powder, thin windows are required on the faces of the specimen holder. By experiment, it was determined that 0.002 inch thick polyester tape added the least artifacts to the signal quality. However, under the weight of the simulant filling the large WR340 waveguide, the tape was distorted and a large, stiff mica window was used instead. Mica also reduces the chance of sample contamination. Also, by experiment, it was determined that filling a $1 / 4 \lambda$ waveguide with

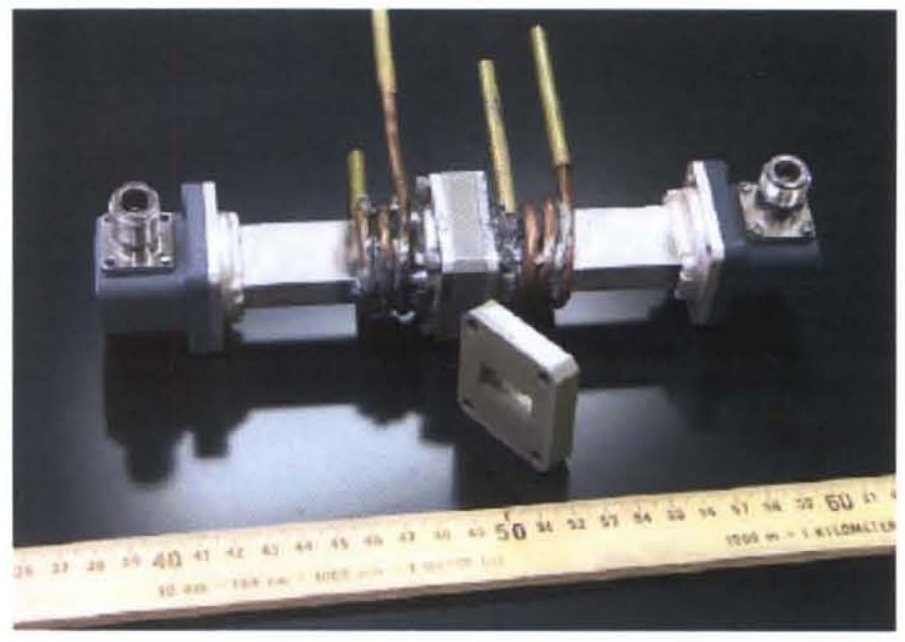

Figure 6. Our custom fabricated $10 \mathrm{GHz}$ (range $8-12 \mathrm{GHz}$ ) waveguide apparatus for dielectric measurements over a range of temperatures, LN2 to above room temperature.

7

American Institute of Aeronautics and Astronautics 092407 
simulant or using a longer path length than $1 / 4 \lambda$ was not optimal for the models to converge.

The best results were obtained by filling the $1 / 8$ $\lambda$ waveguide in a horizontal orientation with only one window on the bottom. Rather than pressing the powder into a pill, the simulant was settled by vibration to approach lunar regolith densities.

A prototype measurement setup for the 2.45 $\mathrm{GHz}$ measurements is shown in Figure 7. Wave guides have been ordered to extend the distance from the coax to waveguide connection to the sample holder.

Our plans are to make measurement at three microwave frequencies, $0.9 \mathrm{GHz}, 2.45 \mathrm{GHz}$ and 10 $\mathrm{GHz}$. The photograph in Figure 8 shows the three waveguides for size comparison.

The original room temperature measurements of the complex permittivity and permeability for JSC$1 \mathrm{~A}$ at 8 to $12 \mathrm{GHz}$ are shown in Figure 8 . Shown are the complex permittivity and complex magnetic permeability. The Agilent, 2-port PM method provided the most useful data including both permittivity and permeability of regolith simulant.

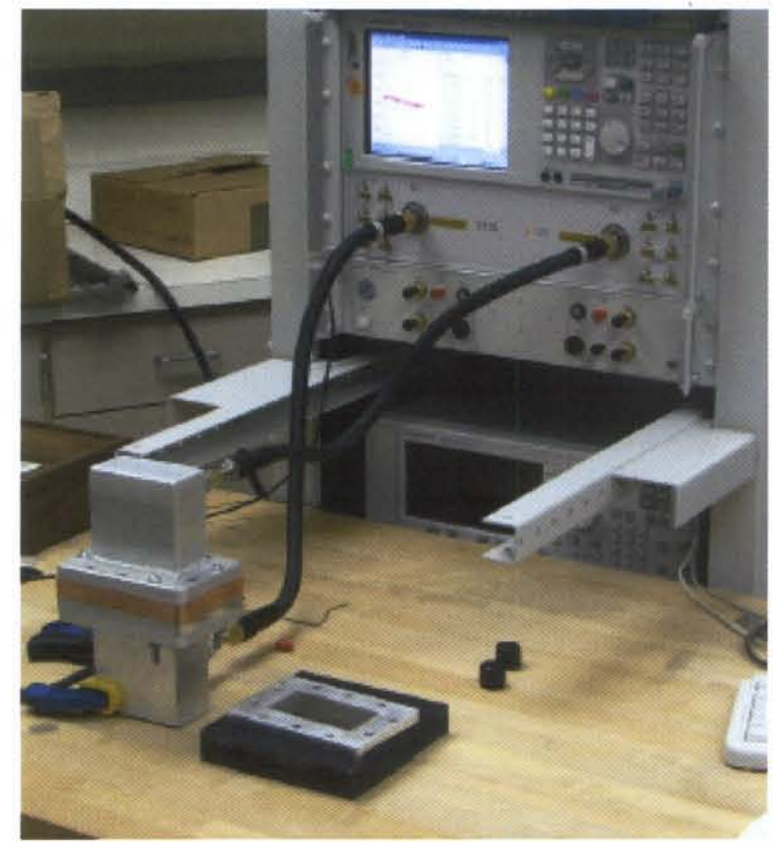

Figure 7. $2.45 \mathrm{GHz}$ materials properties setup used to collect data for Figure 12 below.

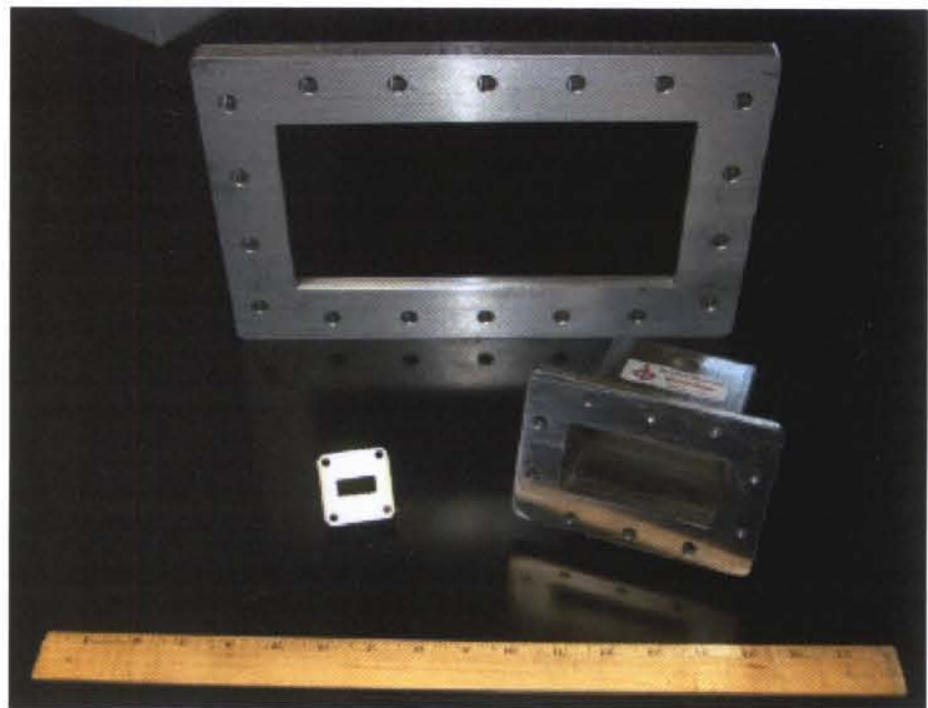

Figure 8. Microwave flanges for the three different microwave frequencies $(0.9 \mathrm{GHz}, 2.45 \mathrm{GHz}$ and $10 \mathrm{GHz})$ used in this project showing the relative sizes of the experimental and test measurement hardware. Their standard sizes are designated WR975, WR340 and WR90 respectively. 
The effect of temperature from -73 to $+43 \mathrm{C}$ on the dielectric constant (real component of permittivity) over the $\mathrm{X}$ band $(8$ to $12 \mathrm{GHz}$ ) for JSC-1A are plotted in figure 10. In general, the dielectric constant increases with increasing temperature and it tends to higher value at higher frequency for temperatures but tends to lower values at higher frequency at lower temperatures. Measurements of the dielectric properties of air over these temperatures show the apparatus expansion or contraction was not a major contributor to the deviations at the high frequencies with temperature.

The effect of temperature on the dielectric properties will significantly affect the penetration of microwaves into the regolith.

Comparison of loss tangent data determined from remote sensing indicate that the values for the lunar soil simulant JSC-1A are slightly more than the Apollo lunar soil. But lunar simulant measurements at lower
JSC1 Dielectric/Magnetic Properties

4-parameter: 4/27/07: $4.16 \mathrm{~mm}$ path

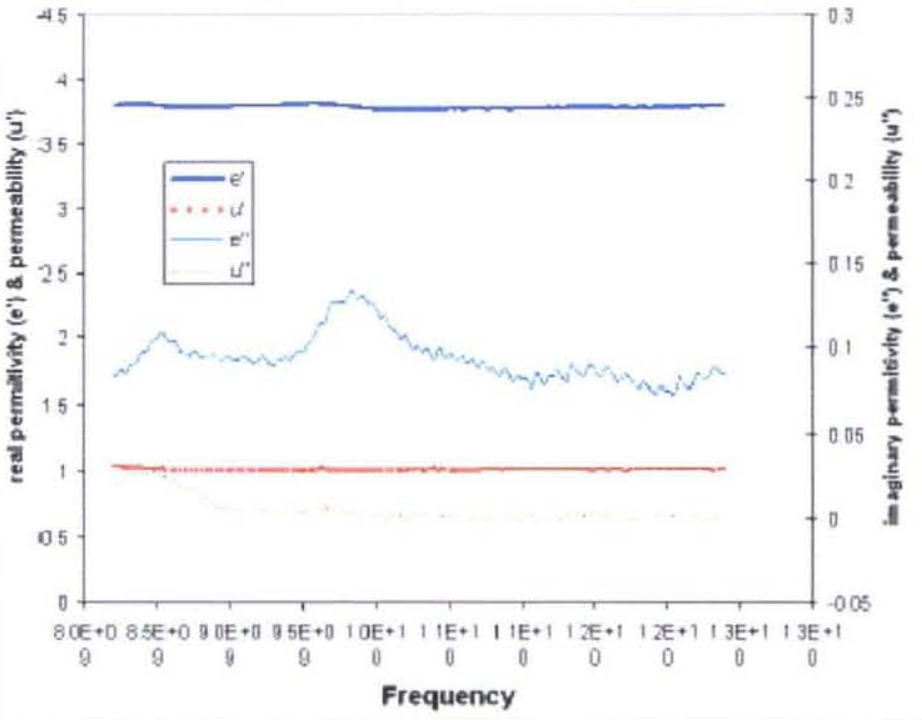

Figure 8. Room temperature measurements of the real and imaginary components of the dielectric constant of JSC1 simulant over the 8-12 $\mathrm{GHz}$ frequency range (X-band).

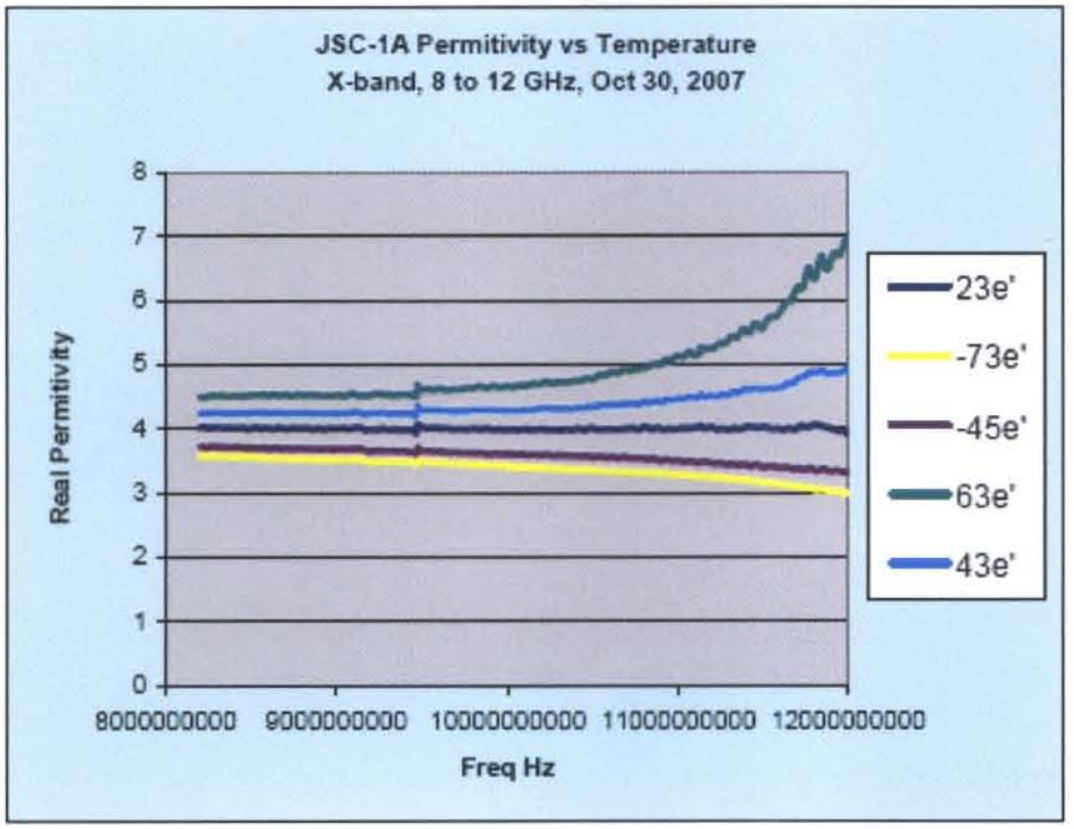

cryogenic temperatures would have lower values more in line with those from the indirect measurements of the moon using ground based measurements from earth during lunar eclipses.

Figure 10. Dielectric constant (real component of permittivity) vs. frequency over the X-band $(8$ to $12 \mathrm{GHz})$ for JSC-1A at various temperatures from $63 \mathrm{C}$ down to $-73 \mathrm{C}$.

\section{Future Work}

Future experiments include scaling up of the lunar test bed facility into a vacuum system. Such a system will permit the testing of microwave penetration into soil and water extraction from a greater depth under more realistic conditions. The facility will require system design, setting up the microwave hardware in a large vacuum facility. Some effort was spent in the current research program designing and testing microwave applicator design as well as other methods for microwave power delivery. Modeling software will permit the calculation of microwave propagation through the launcher and delivery to the regolith. The power distribution and attenuation could be 
calculated for prototype systems. The FEA software COMSOL Multiphysics is being used to determine the heating of soil simulant based on microwave frequency and simulant dielectric properties.

Presently in the process of scaling up our existing Lunar simulation chamber to measure the penetration of microwave energy, the power to extract water from one meter depth, and the measurement of percolation rates from the soil. Microwaves will be beamed in from the top of a vacuum chamber cooled with liquid nitrogen (-150 C) and filled with simulant to a depth of 1 meter. In order to implement these experiments.

It is known that the lunar regolith is highly compacted. Water vapor will have difficulty percalating through the compacted layers. Efficient cold trap design and testing is also needed. There are a couple of design concepts that would be suitable for different extraction hardware configurations.

A proposal is pending with the JSC lunar soil repository, for the measurement of dielectric properties at 2.45 and $10 \mathrm{GHz}$. The comparison of the complex permittivity and complex permeability of Apollo lunar soil samples with lunar regolith simulant will be very valuable for future work and prototype design studies.

In order to plan for future lunar lander science payload experiments, we will examine scaled down low power microwave processing methods. Low power microwave components for preliminary prototyping and testing will need to be identified, procurement, and tested.

Water extraction is not the only process that could employ microwave processing. Many other volatiles can be extracted in exactly the same way. Each would require their own collection schemes since the vapor pressures and chemistries are radically different from water. In our other studies with regolith simulant, it has also been shown that one can sinter or melt the regolith for dust mitigation applications such as outpost entrance pads and for launch/landing pad site preparation. The effectiveness of microwave processing can be better predicted by knowing the dielectric properties of lunar simulants and lunar soil. The measurement of dielectric properties is the second complementary aspect of this project.

\section{Conclusions}

Proof of concept experiments using the 'moon in a bottle' proved that microwave heating of cryogenic lunar regolith in a vacuum can efficiently extract water. One kilowatt of power removed $2 \mathrm{~g}$ of water from $200 \mathrm{~g}$ of regolith simulant at cryogenic temperature. Part of the $1 \mathrm{KW}$ of microwave energy also went to heat the $1 \mathrm{~kg}$ simulant bed. Hardware was procured, configured, and tested for microwave dielectric property measurements at three frequencies 10, 2.45 and $09 . \mathrm{GHz}$. The Agilent network analyzer and software was used to make measurements on JSC-1 and JSC-1A. Microwave permittivity and permeability measurements of simulant at 10 $\mathrm{GHz}$ have been made down to cryogenic temperatures and well above room temperature. A change of permittivity with temperature was observed that could not be accounted for by apparatus thermal expansion. The reduction in permittivity with lower temperature means deeper penetration of the microwaves on the cold moon. The increase in dielectric constant with temperature means that as the regolith warms up during the microwave processing, the coupling to the microwave power will increase and the penetration will decrease. The experimental results from our proposed study will be very useful for trade studies and design of lunar surface operations. Any microwave processing of lunar material would likely use frequencies in the 10 to $0.9 \mathrm{GHz}$ range.

References

1. In Situ Fabrication and Repair and Resource Utilization Final Report for Nondestructive Evaluation Technologies Center Strategic Investment Project ISFR-CSI-004 Baseline, Carole McLemore/MSFC/VP33 Project Manager.

1. Larry Taylor, personal communication.

2. Lunar Regolith Simulant Workshop, Huntsville, AL, 2004

3. Lunar Sourcebook, Lunar and Planetary Institute, 1991

4. Strangway, D.W., Pearce, G.W., and Olhoeft, G.R., 1977, Magnetic and dielectric

properties of lunar samples, in The Soviet-American Conference on the

Cosmochemistry of the Moon and Planets: National Aeronautic and Space

Administration SP-370 p. 417-432.

5. Chung, D. H., Westphal, W. B., and Olhoeft, G. R.; Dielectric properties of Apollo

14 lunar samples; Proc. Third Lunar Science Conference, supplement to

Geochimica et Cosmochimica Acta, v3, pp3161-3172, MIT Press, 1972

6. Bassett, H. L. and Shackelford, R. G.; Dielectric properties of Apollo 14 lunar

samples at microwave and millimeter wavelengths; Proc. Third Lunar Science

Conference, supplement to Geochimica et Cosmochimica Acta, v3, pp3157-

3160, MIT Press, 1972

7. Fisk, M., W McGregor, R. Pope, C. McLemore, R. Kaul, G. Smithers, E. Ethridge 
and H Toutanji, Lunar in situ Materials Based Surface Structure Technology

Development Efforts at MSFC," E07 at STAIF 2007, Feb 2007.

8. Ethridge, E and W. Kaukler, "Microwave Extraction of Water from Lunar Regolith Simulant," E06 STAIF 2007, Feb 2007.

9. Hepburn, F. And Toby Case, unpublished data.

10. Jackson, John David, Classical Electrodynamics, p250,265, John Wiley \& Sons, New York, (1962).

11. Richter, Scott W., "Experimental Determination of In-Situ Utilization of Lunar Regolith for Thermal Energy Storage", NASA CR 191050 (1993).

12. Vartanian, P.H, W.P. Ayres, and AL Helgesson, "Propagation in Dielectric Slab Loaded Rectangular Waveguide", IRE Transactions on Microwave Theory and Techniques 215-222 (1958).

13. Pozar, D, Microwave Engineering, John Wiley \& Sons, pl41 (1988).

14. Personal Communication, On-line Microwave Encyclopedia, microwave101.com 